\title{
A Política Nacional de Educação Permanente em Saúde: percepção dos cirurgiões-dentistas da Atenção Primária à Saúde
}

\author{
Janaína Rocha de Sousa Almeida*; Davi de Oliveira Bizerril**; Katia de Gois Holanda Saldanha***; \\ Franklin Delano Soares Forte****; Maria Eneide Leitão de Almeida***** \\ * Doutora em Clínica Odontológica, Professora, Curso de \\ Odontologia, Centro Universitário Christus \\ ** Doutor em Clínica Odontológica, Professor, Curso de \\ Odontologia, Universidade de Fortaleza \\ *** Mestre em Clínica Odontológica, Professora, Curso de \\ Odontologia, Centro Universitário Christus \\ **** Doutor em Odontologia Preventiva e Social, Professor, \\ Curso de Odontologia, Universidade Federal da Paraíba \\ ***** Doutora em Odontologia Preventiva e Social, Professora, \\ Curso de Odontologia, Universidade Federal do Ceará
}

Recebido em 18/08/2018. Aprovado em 22/09/2019.

\begin{abstract}
RESUMO
Tomando-se como referência a Política Nacional de Educação Permanente em Saúde (PNEPS), que busca a formação dos trabalhadores de acordo com as necessidades da população, gestão e profissionais, o objetivo desse estudo foi conhecer e analisar a percepção do cirurgião-dentista (CD) da Estratégia Saúde da Família (ESF) sobre a PNEPS. Trata-se de um estudo quantitativo descritivo, transversal e observacional, com amostra composta por $173 \mathrm{CD}$ atuantes nas Unidades de Atenção Primária em Saúde do Município de Fortaleza/CE. Os dados foram coletados por meio de questionário semiestruturado. Os dados categóricos foram expressos na forma de frequência absoluta e percentual, sendo analisados por meio do teste qui-quadrado. Avaliando o perfil socioeconômico, a maioria dos entrevistados foi do gênero feminino (74,6\%), casado $(72,8 \%)$, com renda média de 7 a 10 salários mínimos (49,1\%). Quanto à PNEPS, 51,2\% a conheciam parcialmente, sendo a Educação Permanente em Saúde (EPS) avaliada como regular por 48,8\% e como boa por 33,3\%. Destaca-se que a compreensão da EPS como fator contribuidor da formação profissional foi positiva para 97,7\% dos entrevistados. Observou-se que maior participação nas atividades de EPS favorece a avaliação positiva da PNEPS. Essa política ainda é desconhecida por muitos CD da ESF. As atividades de EPS realizadas no município de Fortaleza são consideradas regulares pela maioria dos profissionais entrevistados. Visando avançar com a EPS, os participantes reforçam a importância das ações na perspectiva da reflexão pelo/no trabalho na atenção primária à Saúde, buscando mudanças no processo de trabalho em saúde e consequentemente a melhoria da atenção em saúde prestada à população.
\end{abstract}

Descritores: Educação. Estratégia Saúde da Família. Saúde Bucal. 


\section{INTRODUÇÃO}

Com a implementação do Sistema Único de Saúde (SUS) a política de recursos humanos começou a ser baseada em princípios claros, em que o profissional de saúde deve ser capaz de propiciar uma assistência mais efetiva, equânime e de qualidade ${ }^{1}$. Na Constituição de 1988, o artigo 200 estabelece que é competência do SUS ordenar a formação de recursos humanos na área da saúde ${ }^{2}$. Para isso, algumas estratégias foram implementadas, buscando tornar o SUS uma rede de ensino-aprendizagem.

No início dos anos 1980, a Organização PanAmericana de Saúde lançou a proposta da Educação Permanente em Saúde (EPS), cuja finalidade era orientar o processo de formação dos trabalhadores da saúde ${ }^{3,4}$, a qual deve ser iniciada no decorrer dos cursos de graduação e perpetuada durante toda a vida do profissional.

A Política Nacional de Educação Permanente em Saúde (PNEPS) foi a estratégia lançada pelo Ministério da Saúde (MS) brasileiro, por meio da portaria $\mathrm{n}^{\circ}$ 198/GM/MS em 13 de fevereiro de 2004, para a formação e desenvolvimento de trabalhadores para o setor ${ }^{5}$. A implantação da PNEPS objetivou a formação e o desenvolvimento dos profissionais de saúde, propondo que o processo de formação dos trabalhadores aconteça tomando como referência a necessidade de saúde da população, da gestão e do controle social em saúde ${ }^{4,6}$. Entre seus objetivos destaca-se a construção de uma rede de ensinoaprendizagem no exercício de trabalho no SUS, visando formar e desenvolver trabalhadores, observando sempre as necessidades de saúde dos usuários ${ }^{7}$.

Entre as características da proposta de EPS, a aprendizagem significativa é uma das mais importantes, fundamentada na proposta pedagógica de Paulo Freire $^{8}$, com o trabalho baseado na problematização, utilizando os dados vivenciados no dia a dia dos profissionais e levando em consideração seus conhecimentos e concepções pré-existentes $^{3}$. Portanto, os cenários de prática passam a servir como um espaço de problematização, diálogo, reflexões e construção de consensos, em que seja possível promover um serviço de saúde com mais qualidade ${ }^{3,9}$, que favoreça a formação de profissionais com perfil adequado e capacidade de aprender a aprender, trabalhar em equipe, comunicar-se, refletir criticamente e aprimorar qualidades humanistas ${ }^{10}$.

Seguindo as orientações de organização das atividades de EPS, o município de Fortaleza/CE criou, por meio da Portaria $n^{0} 160 / 2006$ da Secretaria Municipal de Saúde (SMS), o Sistema Municipal Saúde Escola $(\mathrm{SMSE})^{11}$ com o propósito de fortalecer a ideia de um sistema integrado de ensino, serviço e pesquisa. Esse sistema destaca-se por sua amplitude, envolvendo instituições de ensino superior e técnico, públicas e privadas e todas as unidades de saúde, o que torna a experiência uma das mais amplas do país ${ }^{12}$, caracterizando-se por ofertar cursos de aperfeiçoamento dos serviços e treinamento dos profissionais. Em 2013, o SMSE passou a ser chamado de Coordenadoria da Gestão do Trabalho e Educação em Saúde (COGTES), reafirmando a importância de trabalhar a EPS no município.

Diante dessas considerações e compreendendo a relevância da educação no trabalho para a transformação dos serviços de saúde, realizou-se o presente estudo, pioneiro no município de Fortaleza/CE, com o objetivo de conhecer e analisar a percepção dos CD da Estratégia Saúde da Família (ESF) sobre a PNEPS.

\section{MÉTODOS}

Trata-se de uma investigação
epidemiológica transversal, quantitativa,
descritiva, observacional, pois analisa os dados por
meio da observação sistemática de suas
características, sem intervenção nas variáveis ${ }^{13}$.
O estudo teve como cenário, o município de


Fortaleza, capital do Estado do Ceará, localizado na região Nordeste do Brasil, com 108 Unidades de Atenção Primária em Saúde (UAPS), divididas em seis Secretarias Regionais de acordo com as características e semelhanças da sua população e território. Destaca-se que o município de Fortaleza é a quinta maior capital do Brasil, possuindo população de 2.447.409 habitantes, apresentando uma Rede de Atenção à Saúde organizada a partir da Atenção Primária à Saúde (APS) ${ }^{11}$.

A população-alvo desta pesquisa foi composta pelos CD da ESF do município. Para a definição do tamanho da amostra foram considerados erro amostral de 5\%, nível de confiança de $95 \%$ e percentual de observação (p) de $50 \%$, ápice de confiança estatística para cálculos amostrais de dados binários, quando a frequência da variável de estudo é desconhecida. Considerando a população de $284 \mathrm{CD}$, de acordo com o Sistema de Informação de Atenção Básica (SIAB) em fevereiro de 2014, chegou-se a um tamanho amostral de 164. Considerando a possibilidade de eventuais perdas, foram avaliados $173 \mathrm{CD}$.

$\mathrm{O}$ critério de inclusão foi que o $\mathrm{CD}$ deveria estar atuando na ESF há pelo menos dois anos. Foram excluídos os profissionais que estavam afastados das suas funções na ESF por estarem atuando na gestão, atenção secundária, devido a férias ou licença no momento da coleta dos dados. Também foram excluídos os participantes que não responderam a pelo menos $70 \%$ das questões do instrumento de pesquisa.

Os dados foram coletados por meio de um questionário semiestruturado, aplicado aos $\mathrm{CD}$ da ESF por duas pesquisadoras. Previamente, as pesquisadoras discutiram sobre o objeto do estudo e o instrumento de coleta de dados, a fim de alinhar a condução dos trabalhos. O questionário foi validado por uma equipe de especialistas no assunto, e posteriormente submetido a teste piloto para a verificação de possíveis alterações necessárias. Os questionários do estudo piloto tiveram seus dados descartados dos resultados finais da pesquisa.

Os profissionais foram entrevistados nas UAPS em que atuavam ou em reuniões de equipes realizadas pela Secretaria Municipal de Saúde. A coleta de dados ocorreu de agosto de 2014 a agosto de 2015.

O questionário foi dividido em duas partes: a primeira, com 13 questões sobre a identificação dos profissionais, e a segunda contendo 14 questões referentes às percepções sobre a EPS. Os dados foram tabulados no Microsoft Excel® e exportados para o software Statistical Packcage for the Social Sciences (SPSS) versão 17.0 para Windows. Os dados categóricos foram expressos em forma de frequência absoluta e percentual e analisados por meio do teste qui-quadrado. Os dados numéricos foram expressos em forma de média \pm desviopadrão e analisados por meio do teste de KruskallWallis (dados não paramétricos, teste de Kolmogorov-Smirnov).

Foi considerado um índice de confiança de 95\% expondo-se as razões de prevalências e respectivos intervalos de confiança das comparações estatisticamente significantes. As variáveis foram distribuídas em frequência e proporção. Realizou-se associações entre as variáveis dependentes "conhecer a PNEPS" e "avaliação da PNEPS" e as demais variáveis.

$\mathrm{O}$ estudo foi realizado conforme as exigências da resolução No 466/2012 do Conselho Nacional de Saúde, com aprovação do projeto pelo comitê de ética em pesquisa da Universidade Federal do Ceará, com o parecer $\mathrm{n}^{\circ} 526.915$ (CAAE 25677613.8.0000.5054).

\section{RESULTADOS}

Foram entrevistados $173 \mathrm{CD}$ da ESF, cuja média de idade foi de 38,8 $\pm 8,6$ anos e tempo de formação de 16 $\pm 7,4$ anos. A maioria dos entrevistados era do gênero feminino $(74,6 \%)$, casada $(72,8 \%)$, com renda média entre 7 e 10 
salários mínimos (49,1\%). Ressalta-se que em 2014 o valor do salário mínimo era $\mathrm{R} \$ 724,00$, estando o dólar com o valor de 2,40 reais; assim, o salário mínimo era equivalente a U\$ 301,66. Em 2015, o salário mínimo era $\mathrm{R} \$ 788,00$, estando o dólar com o valor de 2,69 reais; assim, o salário mínimo apresentou desvalorização e equivalia a U\$ 292,93.

Quanto à localização da regional, 28,9\% trabalhavam na Secretaria Regional VI. Com relação ao vínculo empregatício, a maioria possuía outro emprego (53,8\%), sendo a atuação em consultório próprio exercida por $77,4 \%$ dos participantes.

No que diz respeito à formação de graduação, a maioria a realizou em instituição pública $(67,6 \%)$. Destaca-se que a quase totalidade dos profissionais $(97,1 \%)$ possui curso de pósgraduação, a maioria $(91,3 \%)$ no nível de especialização, sendo $71 \%$ em Clínica Odontológica e $60 \%$ em Saúde Coletiva (tabela 1).

Observa-se que $51,2 \%$ dos participantes conhecem parcialmente a PNEPS. $\mathrm{O}$ fato de a política ter como público-alvo profissionais, usuários e gestores foi destacado por $82 \%$ dos profissionais. Foi ainda reconhecido que a PNEPS tem como objetivos orientar usuários e gestor, fazer atualização técnico-científica e transformação das práticas (67,5\%) (tabela 2).

Quanto à promoção de atividades de EPS, $48,3 \%$ dos entrevistados relataram que foram realizadas, contudo $63,4 \%$ apontaram não existir uma frequência exata de execução. Para $37,9 \%$ dos $\mathrm{CD}$ as atividades deveriam ocorrer com periodicidade mensal. A maioria já participou da EPS $(89 \%)$ e $52,2 \%$ acreditam que as atividades propostas contemplam parcialmente a integração entre a teoria e a prática (tabela 2).

A infraestrutura foi considerada parcialmente adequada para as atividades propostas por $53,5 \%$ dos participantes. A EPS foi avaliada como regular e boa por $48,8 \%$ e $33,3 \%$, respectivamente (tabela 2 ).

A compreensão da EPS como fator contribuidor da formação profissional foi de $97,7 \%$, e a metodologia de exposição dialogada foi sugerida como a mais adequada $(61,6 \%)$, ao passo em que foi relatada como a mais utilizada $(67,3 \%)$ (tabela 2).

Não foi observada associação entre a idade, tempo de formado e tempo de atuação na ESF com a variável dependente "conhecimento da PNEPS". Contudo, verificou-se associação significativa entre esta variável dependente e ter cursado pósgraduação na área de Saúde Coletiva - 83,3\% dos CD que fizeram pós-graduação em Saúde Coletiva relataram conhecer a referida política. Também foi observado que $56,3 \%$ dos profissionais que conhecem a PNEPS compreendem as metodologias ativas como o principal método a ser utilizado no desenvolvimento das atividades de EPS (tabela 3 ).

Ressalta-se que foram agrupadas como pósgraduação em Saúde Coletiva os cursos de Saúde da Família, Saúde Pública, Gestão em Saúde, Regulação e Auditoria e Odontologia Legal. Como pós-graduação em clínica foram agrupadas as áreas de Periodontia, Odontopediatria, Ortodontia, Prótese, Cirurgia, Dentística, Implantodontia, Endodontia, Pacientes Especiais e Radiologia.

Quanto mais atividades de EPS são realizadas, mais positiva é a sua avaliação (tabela 4). Foi verificada, também, associação entre a contribuição da EPS para a formação dos profissionais de saúde e o fato de ser avaliada como ótima ou boa. A totalidade dos profissionais que consideram a EPS como ótima ou boa reconhecem a sua contribuição para a formação profissional (tabela 4). Não foi verificada associação significativa entre a avaliação da EPS e a frequência de realização das atividades ou a indicação de uma metodologia.

Os CD foram questionados sobre quais são as temáticas específicas para dar seguimento às atividades de EPS, e as mais citadas foram a 
Tabela 1. Caracterização socioeconômica dos cirurgiões-dentistas da amostra

\begin{tabular}{|c|c|c|}
\hline Variável & $\mathbf{n}$ & $\%$ \\
\hline \multicolumn{3}{|l|}{ Gênero } \\
\hline Feminino & 129 & $74,6 \%$ \\
\hline Masculino & 44 & $25,4 \%$ \\
\hline \multicolumn{3}{|l|}{ Estado Civil } \\
\hline Solteiro & 28 & $16,2 \%$ \\
\hline Casado & 126 & $72,8 \%$ \\
\hline Divorciado & 9 & $5,2 \%$ \\
\hline Separado & 6 & $3,5 \%$ \\
\hline Outros & 4 & $2,3 \%$ \\
\hline \multicolumn{3}{|c|}{ Renda Mensal (salários mínimos) } \\
\hline Até 7 & 35 & $20,2 \%$ \\
\hline 7 a 10 & 85 & $49,1 \%$ \\
\hline 10 a 15 & 39 & $22,5 \%$ \\
\hline 15 a 20 & 8 & $4,6 \%$ \\
\hline Mais de 20 & 1 & $0,6 \%$ \\
\hline \multicolumn{3}{|c|}{ Secretaria Regional na qual trabalha } \\
\hline $\mathrm{I}$ & 22 & $12,8 \%$ \\
\hline II & 22 & $12,8 \%$ \\
\hline III & 32 & $18,6 \%$ \\
\hline IV & 21 & $12,2 \%$ \\
\hline $\mathrm{V}$ & 26 & $15,1 \%$ \\
\hline VI & 50 & $28,5 \%$ \\
\hline \multicolumn{3}{|l|}{ Possui outro emprego } \\
\hline Sim & 93 & $53,8 \%$ \\
\hline Não & 80 & $46,2 \%$ \\
\hline \multicolumn{3}{|l|}{ Qual emprego } \\
\hline Consultório & 72 & $77,4 \%$ \\
\hline Serviço público & 12 & $12,9 \%$ \\
\hline Outros & 9 & $9,7 \%$ \\
\hline \multicolumn{3}{|l|}{ Instituição de formação } \\
\hline Pública & 117 & $67,6 \%$ \\
\hline Privada & 56 & $32,4 \%$ \\
\hline \multicolumn{3}{|l|}{ Possui pós-graduação } \\
\hline $\operatorname{Sim}$ & 168 & $97,1 \%$ \\
\hline Não & 5 & $2,9 \%$ \\
\hline \multicolumn{3}{|l|}{ Nível da pós-graduação } \\
\hline Especialização & 157 & $91,3 \%$ \\
\hline Aperfeiçoamento & 58 & $33,7 \%$ \\
\hline Mestrado & 21 & $12,2 \%$ \\
\hline Doutorado & 1 & $0,6 \%$ \\
\hline Pós-doutorado & - & - \\
\hline \multicolumn{3}{|l|}{ Área da pós-graduação* } \\
\hline Clínica & 110 & $71,0 \%$ \\
\hline Saúde Coletiva & 93 & $60,0 \%$ \\
\hline
\end{tabular}

* Possibilidade de marcar mais de uma opção 
Tabela 2. Conhecimento sobre a Política Nacional de Educação Permanente em Saúde

\begin{tabular}{|c|c|c|c|}
\hline Variável & & $\mathbf{n}$ & $\%$ \\
\hline \multirow{3}{*}{ Conhece a PNEPS } & Sim & 33 & $19,2 \%$ \\
\hline & Não & 51 & $29,7 \%$ \\
\hline & Parcialmente & 88 & $51,2 \%$ \\
\hline \multirow{5}{*}{ Público alvo da PNEPS } & Profissional de nível superior & 1 & $0,6 \%$ \\
\hline & Profissional de nível médio & 34 & $20,4 \%$ \\
\hline & Todos os profissionais, usuários e gestor & 137 & $82,0 \%$ \\
\hline & Gestor & 9 & $5,4 \%$ \\
\hline & Outros & 2 & $1,2 \%$ \\
\hline \multirow{7}{*}{ Objetivo da PNEPS } & Orientar usuários & 1 & $0,6 \%$ \\
\hline & Orientar gestores & 5 & $3,0 \%$ \\
\hline & Atualização técnica-científica & 38 & $22,5 \%$ \\
\hline & Transformação de práticas & 27 & $16,0 \%$ \\
\hline & Todos os objetivos anteriores & 114 & $67,5 \%$ \\
\hline & Nenhuma das anteriores & - & - \\
\hline & Outros & 1 & $0,6 \%$ \\
\hline \multirow{4}{*}{ O município promove EPS } & Sim & 83 & $48,3 \%$ \\
\hline & Não & 7 & $4,1 \%$ \\
\hline & Parcialmente & 74 & $43,0 \%$ \\
\hline & Não sei & 9 & $5,2 \%$ \\
\hline \multirow{6}{*}{$\begin{array}{l}\text { Frequência atual das } \\
\text { atividades de EPS }\end{array}$} & Mensal & 6 & $3,9 \%$ \\
\hline & Trimestral & 3 & $2,0 \%$ \\
\hline & Semestral & 10 & $6,5 \%$ \\
\hline & Anual & 11 & $7,2 \%$ \\
\hline & Sem frequência exata & 97 & $63,4 \%$ \\
\hline & Não sei & 26 & $17,0 \%$ \\
\hline \multirow{3}{*}{$\begin{array}{l}\text { Frequência ideal para as } \\
\text { atividades de EPS }\end{array}$} & Mensal & 64 & $37,9 \%$ \\
\hline & Trimestral & 39 & $23,1 \%$ \\
\hline & Semestral & 37 & $21,9 \%$ \\
\hline \multirow{4}{*}{$\begin{array}{l}\text { Já participou de atividades de } \\
\text { EPS }\end{array}$} & Sim & 153 & $89,0 \%$ \\
\hline & Não & 11 & $6,4 \%$ \\
\hline & Não lembro & 4 & $2,3 \%$ \\
\hline & Não sei & 4 & $2,3 \%$ \\
\hline \multirow{3}{*}{$\begin{array}{l}\text { Existe integração entre teoria e } \\
\text { prática nas atividades de EPS }\end{array}$} & Sim & 52 & $32,7 \%$ \\
\hline & Não & 24 & $15,1 \%$ \\
\hline & Parcialmente & 83 & $52,2 \%$ \\
\hline \multirow{4}{*}{$\begin{array}{l}\text { Infraestrutura adequada para } \\
\text { realizar as atividades de EPS }\end{array}$} & Sim & 49 & $34,0 \%$ \\
\hline & Não & 17 & $11,8 \%$ \\
\hline & Não sei & 1 & $0,7 \%$ \\
\hline & Parcialmente & 77 & $53,5 \%$ \\
\hline \multirow{5}{*}{ Avaliação da EPS } & Ótima & 7 & $4,3 \%$ \\
\hline & Boa & 54 & $33,3 \%$ \\
\hline & Regular & 79 & $48,8 \%$ \\
\hline & Ruim & 19 & $11,7 \%$ \\
\hline & Péssima & 3 & $1,9 \%$ \\
\hline \multirow{2}{*}{$\begin{array}{l}\text { Contribuição da EPS na } \\
\text { formação profissional }\end{array}$} & Sim & 168 & $97,7 \%$ \\
\hline & Não & 5 & $2,3 \%$ \\
\hline \multirow{4}{*}{$\begin{array}{l}\text { Estratégia metodológica } \\
\text { adequada sugerida pelos } \\
\text { entrevistados* }\end{array}$} & Ativa & 57 & $34,8 \%$ \\
\hline & Expositiva & 21 & $12,8 \%$ \\
\hline & Expositiva e dialogada & 101 & $61,6 \%$ \\
\hline & Outras & 14 & $8,5 \%$ \\
\hline \multirow{3}{*}{$\begin{array}{l}\text { Estratégia metodológica } \\
\text { utilizada na EPS* }\end{array}$} & Ativa & 25 & $15,4 \%$ \\
\hline & Expositiva & 68 & $42,0 \%$ \\
\hline & Expositiva dialogada & 109 & $67,3 \%$ \\
\hline
\end{tabular}

\footnotetext{
* Possibilidade de marcar mais de uma opção
} 
Tabela 3. Associação entre o conhecimento da Política Nacional de Educação Permanente em Saúde e as demais variáveis

\begin{tabular}{|c|c|c|c|c|c|c|c|}
\hline & \multicolumn{6}{|c|}{ Conhecimento sobre a PNEPS } & \multirow[b]{2}{*}{ p-valor } \\
\hline & \multicolumn{2}{|c|}{ Sim } & \multicolumn{2}{|c|}{ Não } & \multicolumn{2}{|c|}{ Parcial } & \\
\hline Idade (anos) & \multicolumn{2}{|c|}{$43,2( \pm 8,9)$} & \multicolumn{2}{|c|}{$36,8( \pm 9,6)$} & \multicolumn{2}{|c|}{$38,2( \pm 7,3)$} & $0,686^{\mathrm{a}}$ \\
\hline Tempo de formado (anos) & \multicolumn{2}{|c|}{$19,3( \pm 8,0)$} & \multicolumn{2}{|c|}{$15,4( \pm 6,6)$} & \multicolumn{2}{|c|}{$15,0( \pm 7,3)$} & $0,944^{\mathrm{a}}$ \\
\hline Tempo na ESF (anos) & \multicolumn{2}{|c|}{$8,9( \pm 3,6)$} & \multicolumn{2}{|c|}{$7,8( \pm 3,8)$} & \multicolumn{2}{|c|}{$8,6( \pm 4,5)$} & $0,191^{\mathrm{a}}$ \\
\hline Ter pós-graduação & 33 & $100,0 \%$ & 50 & $98,0 \%$ & 85 & $96,6 \%$ & $0,530^{\mathrm{b}}$ \\
\hline \multicolumn{8}{|l|}{ Tipo de pós-graduação } \\
\hline Especialização & 30 & $90,9 \%$ & 47 & $92,2 \%$ & 80 & $90,9 \%$ & $0,966^{\mathrm{b}}$ \\
\hline Aperfeiçoamento & 10 & $30,3 \%$ & 18 & $35,3 \%$ & 30 & $34,1 \%$ & $0,889^{\mathrm{b}}$ \\
\hline Mestrado & 7 & $21,2 \%$ & 3 & $5,9 \%$ & 11 & $12,5 \%$ & $0,110^{\mathrm{b}}$ \\
\hline Doutorado & - & - & 1 & $2,0 \%$ & - & - & $0,303^{b}$ \\
\hline Pós-doutorado & - & - & - & - & - & - & $1,000^{\mathrm{b}}$ \\
\hline \multicolumn{8}{|l|}{ Área de pós-graduação } \\
\hline Clínica & 13 & $43,3 \%$ & $37 *$ & $82,2 \%$ & $60^{*}$ & $75,0 \%$ & $* 0,001^{\mathrm{b}}$ \\
\hline Saúde Coletiva & $25^{*}$ & $83,3 \%$ & 23 & $51,1 \%$ & 45 & $56,3 \%$ & $* 0,013^{\mathrm{b}}$ \\
\hline \multicolumn{8}{|l|}{ Público alvo da PNEPS } \\
\hline Nível superior & 1 & $3,1 \%$ & - & - & - & - & $0,120^{\mathrm{b}}$ \\
\hline Nível médio & 9 & $28,1 \%$ & 8 & $17,0 \%$ & 17 & $19,3 \%$ & $0,456^{\mathrm{b}}$ \\
\hline Profissionais, usuários e gestores & 26 & $81,3 \%$ & 41 & $87,2 \%$ & 70 & $79,5 \%$ & $0,536^{\mathrm{b}}$ \\
\hline Gestores & 1 & $3,1 \%$ & 2 & $4,3 \%$ & 6 & $6,8 \%$ & $0,673^{\mathrm{b}}$ \\
\hline Outros & 1 & $3,1 \%$ & - & - & 1 & $1,1 \%$ & $0,454^{\mathrm{b}}$ \\
\hline \multicolumn{8}{|l|}{ Objetivo da PNEPS } \\
\hline Orientar os usuários & 1 & $3,0 \%$ & - & - & - & - & $0,126^{\mathrm{b}}$ \\
\hline Orientar os gestores & 0 & $0,0 \%$ & 2 & $4,2 \%$ & 3 & $3,4 \%$ & $0,519^{b}$ \\
\hline Atualização técnico-científica & 6 & $18,2 \%$ & 10 & $20,8 \%$ & 22 & $25,0 \%$ & $0,689^{b}$ \\
\hline Transformar práticas & 6 & $18,2 \%$ & 6 & $12,5 \%$ & 15 & $17,0 \%$ & $0,731^{\mathrm{b}}$ \\
\hline Todos & 22 & $66,7 \%$ & 34 & $70,8 \%$ & 58 & $65,9 \%$ & $0,837^{\mathrm{b}}$ \\
\hline Nenhuma & - & - & - & - & - & - & $1,000^{\mathrm{b}}$ \\
\hline Outros & - & - & 1 & $2,1 \%$ & - & - & $0,281^{\mathrm{b}}$ \\
\hline $\begin{array}{l}\text { Contribuição da EPS na formação } \\
\text { professional (Sim) }\end{array}$ & 32 & $97,0 \%$ & 48 & $98,0 \%$ & 86 & $97,7 \%$ & $0,956^{\mathrm{b}}$ \\
\hline \multicolumn{8}{|c|}{ Estratégia metodológica adequada sugerida pelos entrevistados } \\
\hline Ativa & $18^{*}$ & $56,3 \%$ & 11 & $24,4 \%$ & 28 & $32,9 \%$ & $* 0,013^{\mathrm{b}}$ \\
\hline Expositiva & 2 & $6,3 \%$ & 8 & $17,8 \%$ & 11 & $12,9 \%$ & $0,332^{\mathrm{b}}$ \\
\hline Expositiva dialogada & 14 & $43,8 \%$ & 27 & $60,0 \%$ & 58 & $68,2 \%$ & $0,052^{\mathrm{b}}$ \\
\hline Outros & 2 & $6,3 \%$ & 4 & $8,9 \%$ & 8 & $9,4 \%$ & $0,861^{\mathrm{b}}$ \\
\hline \multicolumn{8}{|l|}{ Estratégia metodológica utilizada na EPS } \\
\hline Ativa & 8 & $25,0 \%$ & 8 & $17,8 \%$ & 9 & $10,8 \%$ & $0,155^{\mathrm{b}}$ \\
\hline Expositiva & 18 & $56,3 \%$ & 18 & $40,0 \%$ & 32 & $38,6 \%$ & $0,210^{\mathrm{b}}$ \\
\hline Expositiva dialogada & 18 & $56,3 \%$ & 30 & $66,7 \%$ & 60 & $72,3 \%$ & $0,256^{\mathrm{b}}$ \\
\hline Outras & 3 & $9,4 \%$ & 0 & $0,0 \%$ & 2 & $2,4 \%$ & $0,057^{\mathrm{b}}$ \\
\hline
\end{tabular}

$\mathrm{p}<0.05$, Kruskal-Wallis / Dunn; Bp $<0.05$, teste qui-quadrado; $* \mathrm{p}<0.05 \mathrm{x}$ demais grupos 
Tabela 4. Associação entre a avaliação da Educação Permanente do Município de Fortaleza e as demais variáveis

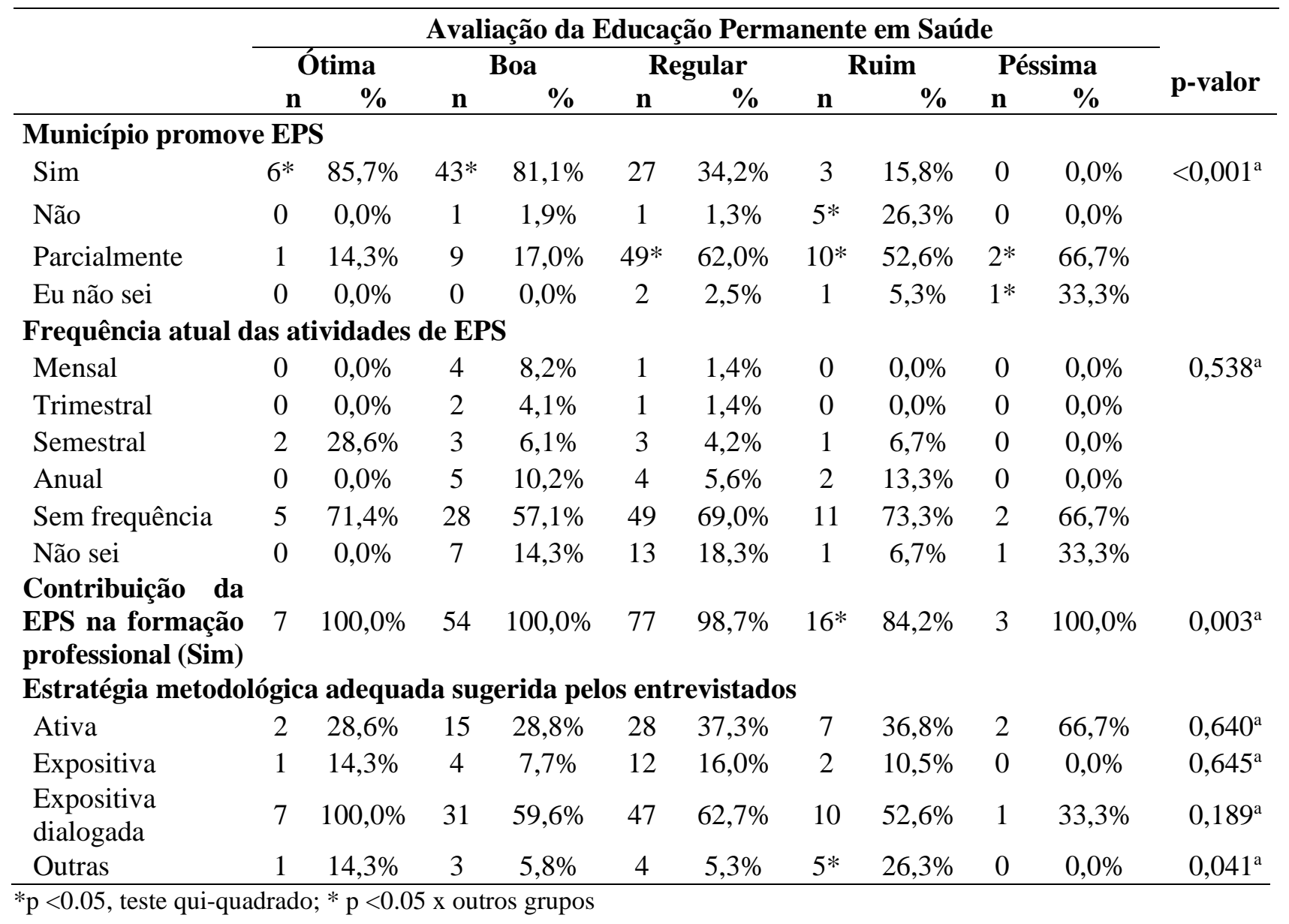

atualização profissional em Urgências Odontológicas com Suporte Básico de Vida, Pacientes Especiais, Farmacologia, Educação em Saúde, Vigilância em Saúde, Saúde Mental e ESF.

\section{DISCUSSÃO}

A maioria dos entrevistados foi do sexo feminino, o que corrobora o estudo de Matos, Toassi e Oliveira ${ }^{14}$ em 2013, que apresenta a feminização das profissões da área da saúde, como um franco crescimento da população feminina em profissões antes desempenhadas pelos homens, tais como a Medicina e Odontologia. O fato da maioria dos entrevistados atuarem na regional VI justificase por ser esta a que possui o maior número de profissionais nas equipes de saúde bucal.
Destaca-se que mais da metade dos entrevistados tinha, além da atuação na ESF, outra fonte de renda, sendo que $77,4 \%$ atuavam também em consultório, o que justifica um elevado percentual de profissionais com cursos de pósgraduação em áreas clínicas.

Os dados aqui apresentados corroboram outro estudo $^{15}$ que define a Odontologia como uma profissão com grande foco na atuação privada e exigência técnica. Contudo, as necessidades de formação foram se modificando ao longo dos anos e atualmente é perceptível a necessidade da atuação profissional no serviço público, onde além da técnica outras competências e habilidades - como a humanização e a capacidade de trabalhar em equipe - são necessárias. 
Observou-se que a PNEPS ainda é desconhecida por muitos trabalhadores do SUS, apesar de publicada em 2004. Tesser et $_{\text {al }}{ }^{9}$ também verificaram desconhecimento pela maioria dos profissionais. Destaca-se que a EPS é uma política nacional para a formação e desenvolvimento dos trabalhadores da saúde, possibilitando o desenvolvimento da educação, assim como a ampliação da capacidade resolutiva dos serviços ${ }^{4}$. Apesar de desconhecerem detalhes sobre a referida política, os $\mathrm{CD}$ compreendem seus objetivos e conhecem seu público-alvo, o que é muito positivo para seu fortalecimento.

Foi reconhecido que o município promove atividades de EPS, porém a falta de frequência das atividades fragiliza o processo de educação. Miccas e Batista em $2014^{16}$ destacaram que a EPS deve ter um caráter contínuo, diferente da Educação Continuada, que está baseada em ações de caráter pontual e fragmentadas, utilizando metodologia tradicional de ensino, características que podem dificultar a aprendizagem.

Destaca-se que o município de Fortaleza proporciona aos profissionais da ESF 8h semanais de sua carga horária de 40h semanais destinadas ao desenvolvimento de atividades de EPS, contudo percebe-se a necessidade de maior regularidade das mesmas. Quase 90\% dos CD já participaram de atividades de EPS, destacando a importância da aprendizagem ter significado. No momento em que as atividades passam a ter associação com as ações diárias dos serviços as atividades tornam-se mais efetivas $^{3}$. Ressalta-se, ainda, que a metodologia tradicional dificilmente proporcionará a integração entre teoria e prática, sendo as metodologias ativas as mais indicadas na busca da aprendizagem significativa ${ }^{17,18}$

A utilização de metodologias problematizadoras com discussões sobre as demandas do território, bem como as necessidades dos profissionais e dos usuários deve ser utilizada nas atividades de EPS. A Portaria ${ }^{\circ} 278$ GM-MS, de 27 de fevereiro de 2014, afirma que uma diretriz da PNEPS é a promoção da aprendizagem significativa por meio da adoção de metodologias ativas e $\operatorname{críticas}^{19}$. A realização de cursos tradicionais que não consideram a aprendizagemtrabalho ou o contexto do local, não agregam valor ao cotidiano dos serviços ${ }^{20}$. Contudo, ainda é possível observar a utilização desse tipo de metodologia em vários serviços ${ }^{16}$.

A metodologia ativa busca trabalhar com problemas para o desenvolvimento dos processos de ensino-aprendizagem, valorizando o aprender a aprender ${ }^{17}$. Apesar da evidência científica ${ }^{17}$ sobre a grande efetividade do trabalho de EPS por meio de metodologias ativas, a presente pesquisa constatou que os CD ainda compreendem as metodologias tradicionais como a melhor opção para a realização das atividades de EPS, fato que pode ser justificado conclusão da graduação até 2000 pela maioria dos profissionais, quando predominavam as metodologias tradicionais.

Para que as atividades de EPS aconteçam de forma satisfatória, além da utilização de uma metodologia apropriada deve existir uma condição física e estrutural adequada, com local específico para seu desenvolvimento. Contudo, evidenciou-se uma fragilidade do município na questão de infraestrutura para o desenvolvimento das atividades de EPS. Considera-se importante que o município de Fortaleza proporcione estrutura física e equipamentos adequados para desenvolver atividades com qualidade para educandos e educadores.

A maioria dos entrevistados classificou a EPS do município como regular, o que deve servir como um alerta para que os gestores verifiquem as principais fragilidades e ressaltem os pontos positivos, buscando elevar o grau de satisfação dos profissionais. A EPS é considerada como fator contribuidor para a formação profissional, elevando desta maneira a qualidade dos serviços. Corroboram essa afirmação Montanha e Peduzzi ${ }^{3}$, 
que destacam a implantação do SUS com base da concepção de integralidade da saúde, gestão participativa e do trabalho em equipe, enfatizando a formação dos trabalhadores como condição essencial para a construção de um serviço de qualidade para a população.

Observou-se associação significativa entre o fato de os profissionais terem pós-graduação em Saúde Coletiva e considerarem as metodologias ativas como as mais adequadas para a realização de atividades de EPS. Os profissionais com formação nesta área têm mais conhecimento sobre a PNEPS, pela ênfase às discussões sobre essa temática, destacando-se que muitos cursos são viabilizados por meio de ações propiciadas pela referida política $^{21}$.

A maioria dos CD acredita que as metodologias ativas são as mais adequadas pois conhecem a PNEPS. Portanto, a metodologia ativa é uma concepção educativa que estimula processos de reflexão, em que o aluno apresenta uma postura ativa e corresponsável em relação a seu aprendizado $^{3,18,22}$. Ressalta-se que a metodologia expositiva dialogada pode ser utilizada como uma metodologia ativa, desde que o educador e os educandos a tornem de fato dialogada.

Ressalta-se ainda que existe uma confusão entre a EPS, que trabalha a aprendizagem significativa, centrada no cotidiano do processo de trabalho, destacando a interdisciplinaridade $\mathrm{e}$ utilizando as metodologias ativas para o seu desenvolvimento, e a Educação Continuada, caracterizada pela transmissão de conhecimentos pontuais e fragmentadas com enfoque nas categorias profissionais ${ }^{9,23}$.

Ao se associar a avaliação da EPS em Fortaleza/CE com as demais variáveis, observouse associação estatística com o município promover atividades de EPS, a contribuição da EPS na formação dos profissionais e a escolha da metodologia adequada.

Sugere-se que a realização de mais atividades de EPS proporcionará uma melhor vivência sobre a PNEPS aos profissionais, o que influencia na avaliação positiva sobre a mesma. Para Lino et al. ${ }^{24}$, a EPS deve fazer parte do fazer e do pensar dos trabalhadores de saúde, proporcionando o crescimento profissional $\mathrm{e}$ pessoal destes, bem como contribuindo para a organização dos processos de trabalho, por meio da problematização da realidade. A realização de atividades de EPS é fundamental para a execução de serviços de saúde com mais eficiência ${ }^{25}$.

A compreensão da importância da EPS para a formação profissional propicia uma avaliação positiva da mesma, sendo possível vislumbrar quão importante é a articulação entre ambas. No estudo de Mendonça et $a l^{22}$ observou-se que a compreensão sobre a EPS permitiu que os profissionais percebessem a importância da política no processo de trabalho, possibilitando a visualização de caminhos para desenvolvê-la.

A discussão sobre a utilização da metodologia adequada necessita ser ampliada entre os trabalhadores, gestores e população. A utilização de uma metodologia inadequada poderá comprometer o processo de EPS e contribuir de forma negativa na avaliação desta EPS. Sugere-se a metodologia ativa problematizadora como a mais adequada, com ela os conhecimentos prévios dos educandos são considerados e o processo de ensino-aprendizagem acontece de forma significativa, o que favorece a implantação de um serviço de saúde de qualidade ${ }^{3,8,9}$.

Finalmente, quanto às percepções dos profissionais sobre as necessidades de atividades de EPS, diversos fatores podem influenciar, tais como interesse especial por uma tarefa em particular, interesse em continuar a aprender, alguma deficiência em sua formação anterior, sua satisfação com a gestão, entre outros ${ }^{25-27}$. É preciso avaliar as necessidades dos trabalhadores, levandose em consideração as necessidades dos gestores e da população, buscando associá-las entre si e 
assim, a EPS se tornará mais efetiva na construção dos serviços de saúde.

\section{CONCLUSÃO}

A PNEPS ainda é desconhecida por muitos cirurgiões-dentistas da ESF. Contudo, a maioria dos profissionais compreende a importância da referida política para a formação dos profissionais do Sistema Único de Saúde. As atividades de EPS realizadas no município de Fortaleza são consideradas regulares pela maioria dos profissionais entrevistados.

Visando avançar com a EPS no município investigado, os participantes da pesquisa reforçam a importância das ações de EPS na perspectiva da reflexão pelo/no trabalho na atenção primária à Saúde, buscando mudanças no processo de trabalho em saúde e consequentemente a melhoria da atenção em saúde prestada à população.

É relevante que os gestores possam juntamente com as equipes ESF planejar atividades de EPS, tendo as metodologias ativas como ferramenta para o desenvolvimento da EPS, reconhecendo-se nesse processo o protagonismo dos trabalhadores, assim como do gestor e da comunidade.

\section{AGRADECIMENTOS}

Agradecemos ao $\mathrm{CNPq}$ por financiar a pesquisa, bem como ao Programa Pró-Ensino na Saúde pela bolsa concedida aos pesquisadores.

\section{ABSTRACT \\ The National Permanent Health Education Policy: dental surgeons' perception of Primary Health Care}

Taking as a reference the National Permanent Health Education Policy (NPHEP), which seeks the training of workers according to the needs of the population, management and professionals, the objective of this study was to know and analyze the perception of the dental surgeon (DS) of the Family Health Strategy (FHS) on NPHEP.
This is a descriptive, cross-sectional and observational quantitative study, with a sample composed of 173 DS active on Primary Health Care Unities from the City of Fortaleza/CE. The data were collected by means of a semistructured questionnaire. The categorical data were expressed as absolute and percentage frequencies, and were analyzed by means of the chi-square test. Evaluating the socioeconomic profile, the majority of the respondents were women $(74.6 \%)$, married $(72.8 \%)$, with an average income of 7 to 10 minimum wages (49.1\%). As for the NPHEP, 51.2\% knew it partially, and Permanent Health Education (PHE) was evaluated as regular by $48.8 \%$ and as good by $33.3 \%$. It is noteworthy that the understanding of PHE as a contributing factor of professional training was positive for $97.7 \%$ of respondents. It was observed that greater participation in the activities of PHE favors the positive evaluation of the NPHEP. This policy is still unknown by many DS of the FHS. The PHE activities carried out in the city of Fortaleza are considered regular by most of the professionals interviewed. Aiming to advance with the PHE, the participants reinforce the importance of actions in the perspective of reflection by/at work in primary health care, seeking changes in the work process in health and consequently the improvement of health care provided to the population.

Descriptors: Education. Family Health Strategy. Oral Health.

\section{REFERÊNCIAS}

1. Pinto ICM, Teixeira CE. Formulação da política de gestão do trabalho e da educação na saúde: o caso da Secretaria Estadual de Saúde da Bahia, Brasil, 2007-2008. Cad Saúde Pública. 2011; 27(9):1777-88.

2. Brasil. Constituição da República Federativa do Brasil. 1988.

3. Montanha D, Peduzzi M. Educação permanente em enfermagem: levantamento de necessidades e resultados esperados 
segundo a concepção dos trabalhadores. Rev Esc Enferm USP. 2010; 44(3):597-604.

4. Peduzzi M, Guerra DAD, Braga CP, Lucena FS, Silva JAM. Atividades educativas de trabalhadores da atenção primária: concepções de educação permanente e de educação continuada em saúde presentes no cotidiano de Unidades Básicas de Saúde em São Paulo. Interface (Botucatu). 2009; 13(30):121-34.

5. Brasil. Ministério da Saúde. Portaria n.198GM, 13 de fevereiro de 2004. Institui a Política Nacional de Educação Permanente em Saúde como estratégia do Sistema Único de Saúde para a formação e o desenvolvimento de trabalhadores para o setor e dá outras providências. Brasília. Ministério da Saúde, 2004.

6. Ferraz F, Backes VMS, Mercado-Martinez FJ, Feuerwerker LCM, Lino MM. Gestão de recursos financeiros da educação permanente em saúde: desafio das comissões de integração ensino-serviço. Cien Saúde Colet. 2013; 18(6):1683-93.

7. Tronchin DMR, Mira VL, Peduzzi M, Ciampone MHT, et al. Educação permanente de profissionais de saúde em instituições públicas hospitalares. Rev Esc Enferm USP. 2009; 43(esp. 2):1210-5.

8. Freire P. Educação como prática da liberdade. $23^{\mathrm{a}}$ ed. Rio de Janeiro: Paz e Terra. 1999.

9. Tesser CD, Garcia AV, Vendruscolo C, Argenta CE. Estratégia Saúde da Família e análise da realidade social: subsídios para políticas de promoção de saúde e educação permanente. Cienc Saúde Colet. 2011; 16(11):4295-306.

10. Frenck J, Chen L, Bhutta ZA, Crisp N, Evans T, Fineberg H. Health profissionals for a new century: transforming education to strengthen helth systems in an interdependent world. Lancet. 2010; 379: 1923-57.

11. Fortaleza. Secretaria Municipal de Saúde. Portaria $n^{\circ}$. 160-2006. Institui o Sistema Municipal Saúde Escola. Fortaleza. 2006.

12. Ellery AEL, Bosi MLM, Loiola FA. Integração ensino, pesquisa e serviços em saúde: antecedentes e iniciativas. Saúde Soc. 2013; 22(1):187-98.

13. Rouquayrol MZ, Silva MGCR. Epidemiologia \& saúde. $7^{\text {a }}$. ed. Rio de Janeiro: MedBook, 2013.

14. Matos IB, Toassi RFC, Oliveira MC. Profissões e ocupações de saúde e o processo de feminização: tendências e implicações. Athenea Digital. 2013; 13(2):239-44.

15. Bockmann FS, Motta BB, Camargo JM, Petry PC, Toassi RFC. O perfil do formando em Odontologia da Universidade Federal do Rio Grande do Sul e expectativas quanto a profissão, 2010-2011. RGO. 2014; 62(3): 267-74.

16. Miccas FL, Batista SHSS. Educação Permanente em Saúde: metassíntese. Rev Saúde Pública. 2014; 48(1):170-85.

17. Marin MJS, Gomes R, Marvulo MML, Primo EM, Barbosa PMK, Druzian S. Pósgraduação multiprofissional em saúde: resultados de experiências utilizando metodologias ativas. Interface (Botucatu). 2010; 14(33):331-44.

18. Simon E, Jezine E, Vasconcelos EM, Ribeiro KSQS. Metodologias ativas de ensino-aprendizagem e educação popular: encontros e desencontros no contexto da formação dos profissionais de saúde. Interface (Botucatu). 2014; 18(12):1355-64.

19. Brasil. Ministério da Saúde. Portaria n. 278, 27 de fevereiro de 2014. Brasília. 2014.

20. Nicoletto SCS, Mendonça FF, Brevilheri ECL, et al. Polos de educação permanente em saúde: uma análise da vivência dos atores 
sociais no norte do Paraná. Interface (Botucatu). 2009; 13(30):209-19.

21. Brasil. Ministério da Saúde. Portaria n.1996GM, 20 de agosto de 2007. Dispõe sobre as diretrizes para a implantação da Política Nacional de Educação Permanente em Saúde e dá outras providências. Diário Oficial da União 2007; 22 ago.

22. Mendonça FF, Nunes EFPA, Garanhani ML, Gonzalez AD. Avaliação de tutores e facilitadores sobre o processo de formação de facilitadores de Educação Permanente em Saúde no município de Londrina, Paraná. Cien Saude Colet. 2010; 15(5):2593-602.

23. Carvalho BG, Turini B, Nunes EFPA, Bandeira IF, Barbosa PFA, Takao TS. Percepção dos médicos sobre o curso de facilitadores de educação permanente em saúde. Rev Bras Educ Méd. 2011, 35(1):132-41.

24. Lino MM, Backes VMS, Ferraz, F, et al. Educação permanente dos serviços públicos de saúde de Florianópolis. Trab Educ Saúde. 2009, 7(1): 115-36.
25. Gaspard J, Yang CM. Training needs assessment in health care professionals in a developing country: the example of Saint Lucia. BMC Medical Education. 2016; 16:112.

26. VanNieuwenborg L, Goossens M, De Lepeleire J, Schoenmakers B. Continuing medical education for general practiotioners: a practice format. Postgrad Med J. 2016; 92(1086): 217-22.

27. Thannhauser J, Russel-Mayhem S, Scott C. Measures of interprofessional education and collaboration. J Interprof Care. 2010; 24(4):336-49.

\section{Correspondência para:}

Janaína Rocha de Sousa Almeida

e-mail: drajanainarocha@hotmail.com

Rua Dr Ratisbona, 171 Fátima

60411-220 Fortaleza/CE 